\title{
Covalent Modification of Graphene Quantum Dots by Fullerene $\mathbf{C}_{60}$ Derivative
}

\author{
Joanna Breczko, Marta Kirmuc, Diana Małgorzata Bobrowska, Krzysztof Winkler \\ University of Bialystok, Institute of Chemistry \\ Ciolkowskiego 1K, Bialystok, Poland \\ j.luszczyn@uwb.edu.pl; m.kirmuc@wp.pl; d.bobrowska@uwb.edu.pl; winkler@uwb.edu.pl
}

\section{Extended Abstract}

Graphene quantum dots (GQDs) are a relatively new class of carbon nanomaterials with a promising potential use. The unique physico-chemical properties of GQDs are intermediate between the features exhibited by nanoparticles, which results from their small size (diameter $<30 \mathrm{~nm}$ ) and features typical for carbon nanostructures with high specific surface area, as the consequence of the fact that GQDs are fragments of a graphene plane [1]. The high reactivity of GQD edges allowing them to be functionalized with oxygen containing groups and to increase their dispersability while good electrochemical properties have been maintained.

Presented results are focused on synthesis of a hybrid system between fullerene $\mathrm{C}_{60}$ and GQDs structures based on covalent modification. In the first step, a 1,3-dipolar cycloaddition reaction to obtain the pyrrolidine fullerene derivative was performed. It involved the introduction of fullerene $\mathrm{C}_{60}$ in the toluene solution containing sarcosine and 4hydroxybenzaldehyde and maintaining the mixture at boiling temperature for $12 \mathrm{~h}$, according to the procedure described in the literature [2]. In parallel, the GQDs modified with carboxylic groups were obtained by ultrasonification of graphite flakes in a nitrating mixture under high temperature conditions [3]. The hydrophilic functional groups, formed mainly at the edges of the GQDs, have reacted with the added thionyl chloride and allow to obtain more reactive acid chloride groups. In the final step, fullerene $\mathrm{C}_{60}$ pyrrolidone was covalently bonded to the functionalized GQDs to form hybrid $\mathrm{C}_{60}-\mathrm{GQDs}_{\text {. }}$

Synthesized $\mathrm{C}_{60} / \mathrm{GQDs}$ material was characterized by microscopic (TEM, SEM) as well as by spectroscopic methods (FTIR, Raman) to confirm each step modifications. According to the combination of GQDs exhibiting large specific capacitance and fullerenes allowing the faradaic processes to occur, obtained hybrid system was expected to act as an interesting charge exchange mediator. Therefore, electrochemical studies were undertaken and the potential of $\mathrm{C}_{60} / \mathrm{GQDs}$ system as an electrode material of the capacitors was examined.

\section{Acknowledgements}

We gratefully acknowledge the financial support of the National Science Centre in Poland (\#2018/02/X/ST5/02958) for J.B.

\section{References}

[1] A. Kelarakis, "Graphene quantum dots: In the crossroad of graphene quantum dots and carbogenic nanoparticles," Curr. Opin. Colloid Interface Sci., vol. 20, pp. 354-361, 2015.

[2] S.K. Das, C.B. Kc, K. Ohkubo, Y. Yamada, S. Fukuzumi, F. D’Souza, "Decorating single layer graphene oxide with electron donor and acceptor molecules for the study of photoinduced electron transfer," Chem. Commun., vol. 49, pp. 2013-2015, 2013.

[3] A. Wolk, M. Rosenthal, S. Neuhaus, K. Huber, K. Brassat, J.K.N. Lindner, R. Grothe, G. Grundmeier, W. Bremser, R. Wilhelm, "A novel lubricant based on covalent functionalized graphene oxide quantum dots," Sci. Rep., vol. 8 pp. 5843(1-9), 2018. 